\title{
Bioresonance, an alternative therapy for mild and moderate depression
}

\author{
DANIELA MURESAN ${ }^{1}$, SEPTIMIU VOIDĂZAN ${ }^{2}$, ANDREEA SALCUDEAN $^{3}$, \\ CRISTINA RALUCA BODO ${ }^{4}$ and IOSIF GABOS GRECU ${ }^{5}$
}

\author{
${ }^{1}$ Doctoral School of Medicine and Pharmacy, Department of Medicine, Departments of ${ }^{2}$ Epidemiology, and \\ ${ }^{3}$ Ethics and Social Sciences, 'George Emil Palade' University of Medicine, Pharmacy, Sciences and Technology; \\ ${ }^{4}$ Psychiatric Clinic No. 2, Mures County Hospital; ${ }^{5}$ Senior Psychiatrist Psychiatric Clinic No. 1, \\ ‘George Emil Palade' University of Medicine, Pharmacy, Sciences and Technology, 540142 Targu Mures, Romania
}

Received November 16, 2021; Accepted December 16, 2021

DOI: 10.3892/etm.2022.11190

\begin{abstract}
Depression is a negative emotional state that may persist for short or long periods of time with varying severity. The aim of the present study was to evaluate the method by which bioresonance therapy can improve the severity of recurrent depressive disorder with moderate and mild episodes experienced by patients. Bioresonance therapy is a method of energy treatment that processes the electromagnetic information of the human body using a sensitive Mora Nova device using electrodes. In addition, this improvement was compared with the one obtained by applying monotherapy with selective serotonin reuptake inhibitors. The study included two groups of patients suffering from depression. The first group received bioresonance treatment for five weeks. The second group received either newly introduced or on-going pharmacological treatment with selective serotonin reuptake inhibitor antidepressants, as monotherapy, for five weeks. An outcome measurement of severity was performed. Results revealed that, the score improvement on the Hamilton Scale, used for assessing depression and comprising 17 items, showed a mean of 3.1 [standard deviation (SD), 1.28] for the bioresonance group one and a mean of $2.2(\mathrm{SD}, 0.61)$ for the second group. The difference between the two data series was statistically significant $(\mathrm{P}<0.0001$, Student's t-test $)$. As the bioresonance therapy outcome was higher than the selective serotonin reuptake inhibitor medication outcome, it can be concluded that bioresonance can reduce the severity of the patients facing recurrent depressive disorder with moderate and mild episodes. Furthermore, the reduction in severity for the bioresonance
\end{abstract}

Correspondence to: Dr Septimiu Voidăzan, Department of Epidemiology, 'George Emil Palade' University of Medicine, Pharmacy, Sciences and Technology, 38 Gheorghe Marinescu Street, 540142 Targu Mures, Romania

E-mail: septi_26_07@yahoo.com

Key words: bioresonance therapy, depression, serotonin, alternative medicine, selective serotonin reuptake inhibitors group compared with the antidepressant medication group was statistically significant.

\section{Introduction}

Depression is the most common disease of affective disorders, which is part of the field of psychiatry. Depression is defined more as a mood disorder than as a thinking disorder, limiting the normal functioning of individuals, and ranging from an extremely mild to a severe form (1).

This negative emotional state can persist for a short period of time or for a longer period, with mild, moderate or severe intensity, which can seriously damage one's health (2). Globally, depression is a leading cause of premature death, along with cardiovascular disease and cancer, limiting the individual from activity, and with a significant risk of suicide (3). Almost 800,000 individuals die each year from suicide (4). Depression is found in 5\% of Europeans and 25\% are likely to experience a depressive episode at least once in their lives (5). By 2030, it is estimated that depression will become one of the most significant mental and behavioral disorders, as it currently occupies a second place in the ranking of diseases worldwide, following cardiovascular diseases (6).

Approximately $80 \%$ of individuals who suffer from a mental disease do not receive treatment, especially in poorly developed countries (7). The most common treatments used in depression are pharmacological medication with antidepressants, or their combination with psychotherapy or psychotherapy as singular treatment. Each employed treatment was found to be useful, but for patients with severe depression, a high dropout rate and low remission were found, and clinically significant differences were observed between antidepressants and placebo. Consequently, patients having mild or moderate depression seek alternative treatments (1).

Bioresonance therapy has been successfully used as an alternative therapy since 1970 in various ailments, by many practitioners worldwide. This type of therapy was presented by physicist Franz Morell and electrical engineering technician Erich Rasche as a result of medical tests in electroacupuncture. Several clinical, physical and organic studies have been completed by global research groups that 
demonstrate the effectiveness of the bioresonance approach in situations such as allergies, rheumatic diseases, respiratory diseases and many painful syndromes (8). In the present study, the aim was to determine whether this new method of therapy is a viable alternative to antidepressants from the selective serotonin reuptake inhibitor class for patients diagnosed with recurrent moderate or mild depressive disorder by decreasing the level of depression quantified with the Hamilton Scale. In addition, the aim was to verify the null hypothesis ( $\mathrm{H} 0)$, which holds that the applied bioresonance therapy does not accelerate the healing process in patients with recurrent moderate and mild depressive disorder, compared with antidepressant medication from the selective serotonin reuptake inhibitor class. The alternative hypothesis (H1) held that the applied therapy accelerates the healing process in patients with recurrent moderate and mild depressive disorder compared with antidepressant medication from the selective serotonin reuptake inhibitor class.

\section{Patients and methods}

General. The present study included patients with recurrent mild and moderate depressive disorder as per the criteria listed in the Diagnostic Manual of Mental Disorders Five (DSMV) (2).

The patients were selected from the Mureș County Clinical Hospital, Psychiatry Clinic I, in Targu-Mures, Romania and in the specialized outpatient clinic and in the Terapia Ultramed Bioresonance Therapy Practice within the Terapia Ultramed Clinic of Targu-Mures. The retrospective study was conducted from October 2017 to 2018. Participants received an explanation on how the research would be carried out, its duration, benefits, stages, implications, data confidentiality and the possibility to exit the study at any point. Participation in the study was based on volunteering. Subjects received an informed consent form regarding their participation in the study, which was knowingly signed by those who agreed. The members of the Ethics Committee of the Mureș County Clinical Hospital approved the study favorably (no. 16462/16.10.2017). The patients who were treated only with bioresonance therapy did not undergo pharmacological treatment with antidepressants.

Patient groups. The analyzed group comprised women and men, from different social backgrounds, aged between 18 and 89 years (mean 49 years/SD 17.16 years). Patients that had undergone suicidal attempts in their medical history, patients with pacemakers and pregnant women were excluded.

The participants were divided into two groups as follows: Group 1 included 40 patients (31 women and 9 men), with 17 females having recurrent mild depressive episodes, and 14 females recurrent moderate depressive episodes. Within the male category, 4 men suffered from a recurrent moderate depressive episode and 5 men from a recurrent mild depressive episode. They received bioresonance therapy solely. Group 2 included 40 patients (19 were women and 21 men). All study participants in this group were previously diagnosed with recurrent moderate depressive episodes. They received a monotherapy treatment with antidepressants from the selective serotonin reuptake inhibitor class. The detailed demographic structure of the two groups is detailed in Table I.
Method. For all patients, the decrease in the level of depression, within a period of maximum 2 months, from the first to the fifth session, was monitored.

The bioresonance treatment consisted of $20 \mathrm{~min}$ and was conducted using a Mora Nova bioresonance device (Med-Tronik GmbH, Germany). The bioresonance device automatically displays on the screen of the device the 16 values of the energy field, eight at the beginning and eight at the end of each bioresonance session. When commencing with the therapy, the conductance between the hands and feet is automatically measured and displayed. The principle of basic bioresonance therapy with the Mora Nora device includes endogenous therapy, by means of hand and foot electrodes, on the palms and fingers and soles, respectively, where the reflexogenic areas of the organs are provided, being considered the fundamental principle of therapy with physical bioresonance. Through these measurements the specific reaction of the patient to the applied therapy was evaluated, showing the mental state in which the patient was before and at the end of each treatment session (9).

The Mora Nova device is an electromagnetic transceiver, with a frequency between 0.1 and $480,000 \mathrm{~Hz}$ insured with a frequency filter from 1 up to $500,000 \mathrm{~Hz}$, which works through connection to a source of electricity (10).

The basic principle of coupled electric oscillators is to consider the cells as complex systems of layers, or as charged structures, respectively, all biomolecules being highly charged ions or even multipoles. Cellular systems are regarded as numerous different distributions of charges and currents, induced by the transfer of charge through hydrogen bonds or other molecular deformations. The advanced technique of the Mora device, through the biorhythm program, separated the oscillating information of the molecules that stress the body from the oscillation information that is conducive to the body's health. The human body incorporates similar discrete frequencies of molecular oscillations, given by the power of intermolecular forces (11). When connecting the patient to the bioresonance device, no mechanical devices such as a smartphone, jewellery, clips or any other electronic device should be present. Before and after each treatment session, the electrodes are cleaned with $70^{\circ}$ alcohol (12).

The therapy was individualized according to the biorhythm of the patient and incorporated high and low potencies. Through biorhythm, the therapeutic program selected from the device software regulated and controlled the patient's own oscillating information, as well as their ability to adjust, in order to initiate precisely target healing processes (13).

Endogenous therapy. Endogenous therapy is included in the principle of basic bioresonance therapy with the Mora Nova device and is considered the fundamental principle of bioresonance therapy at the physical level. This type of therapy involves the destructive inference or the so-called 'overlapping extinction' of rigid, isolated vibrations, considered pathological vibrations in themselves. In this way, they seem to be integrated into the flexible vibrational composition of patients through the processes of self-regulation. The physiological blockages correlated with the 'rigid' vibrations later dissolve. According to these hypotheses, 'pathological vibrations' are correlated with the disease on an electromagnetic plane. A weak electromagnetic interaction has 
Table I. Demographic aspects of the studied groups.

\begin{tabular}{|c|c|c|}
\hline Variable & Group 1 Bioresonance & Group 2 SSRI \\
\hline Sex (female) & $77.50 \%$ & $47.50 \%$ \\
\hline \multicolumn{3}{|l|}{ Age (years) } \\
\hline Mean \pm SD & $43.85 \pm 12.58$ & $55 \pm 17.16$ \\
\hline \multicolumn{3}{|l|}{ Marital status (\%) } \\
\hline Married & 50.00 & 55.00 \\
\hline Divorced & 30.00 & 7.50 \\
\hline Single & 20.00 & 20.00 \\
\hline Widower & 0.00 & 17.50 \\
\hline \multicolumn{3}{|l|}{ Educational level (\%) } \\
\hline Primary & 0.00 & 12.50 \\
\hline Gymnasium & 0.00 & 17.50 \\
\hline High school & 30.00 & 30.00 \\
\hline Secondary & 12.50 & 0.00 \\
\hline Vocational school & 5.00 & 20.00 \\
\hline Higher education & 52.50 & 20.00 \\
\hline \multicolumn{3}{|l|}{ Environment (\%) } \\
\hline Rural & 35.00 & 37.50 \\
\hline Urban & 65.00 & 62.50 \\
\hline
\end{tabular}

SSRI, selective serotonin reuptake inhibitors; SD, standard deviation.

Table II. SSRI medication description.

\begin{tabular}{llr}
\hline Medication & \multicolumn{1}{c}{ Dosage } & No. of patients \\
\hline Escitalopram & $10 \mathrm{mg}$ Once per day & 2 \\
Mirtazapine & $30 \mathrm{mg}$ Once per day & 17 \\
Mirtazapine & $30 \mathrm{mg}$ Half per day & 2 \\
Sertraline & $50 \mathrm{mg}$ Once per day & 16 \\
Sertraline & $50 \mathrm{mg}$ Twice per day & 1 \\
Sertraline & $50 \mathrm{mg}$ Once and a half per day \\
\hline
\end{tabular}

SSRI, selective serotonin reuptake inhibitors.

a physiological consequence, due to the informative catalytic effect, because they are weak interactions, in an initially fragile situation. The energy needed to carry out the program must be provided by the living system itself, through the acupressure points of the lower and upper limbs. Bioresonance therapy also promotes the individual's potential for self-healing (12).

Monotherapy. The second group received monotherapy with selective serotonin reuptake inhibitors (SSRIs) as indicated in Table II. SSRIs are part of one of the most widely used classes of antidepressants, with lower toxicity and much higher safety compared to first-generation antidepressants, which include monoamine oxidase inhibitors and tricyclic antidepressants. Regarding the mechanism of action, SSRIs inhibit serotonin reuptake, leading to an increase in the amount of time serotonin stays in the synaptic cleft, resulting in longer receptor stimulation, leading to greater accumulation of serotonin in the synaptic cleft and increased signalling between synapses. SSRI treatment stimulates $5 \mathrm{HT}_{1 \mathrm{~A}}$ and $5 \mathrm{HT}_{7}$ receptors on cell bodies in the nucleus of the raffia and $5 \mathrm{HT}_{1 \mathrm{D}}$ receptors on serotonergic terminals, reducing 5HT synthesis and release. With repeated administration of an SSRI, there is a gradual decrease and desensitization of these self-receptor mechanisms. Downward regulation of $5 \mathrm{HT}_{2 \mathrm{~A}}$ postsynaptic receptors may contribute either to direct antidepressant efficacy or by influencing noradrenergic function and other serotonergic receptors. Other postsynaptic $5 \mathrm{HT}$ receptors remain receptive to increased synaptic concentrations of 5HT and contribute to the therapeutic effects of SSRIs. The subsequent effects of SSRI treatment may also be important in mediating the final responses of the therapy $(14,15)$.

Hamilton Scale. In order to assess the therapeutic outcome, the Hamilton Scale was used to assess depression composed of 17 items (Ham-D: 17). The Hamilton Scale measures individual 
Table III. Hamilton Scale score between the initial and the final sessions for each group.

\begin{tabular}{lccc}
\hline $\begin{array}{l}\text { Group 1 } \\
\text { Bioresonance }\end{array}$ & $\begin{array}{c}\text { Hamilton score } \\
\text { Initial }\end{array}$ & $\begin{array}{c}\text { Hamilton score } \\
\text { Outcome }\end{array}$ & $\begin{array}{c}\text { Hamilton score } \\
\text { Difference }\end{array}$ \\
\hline Mean & 16.9 & 13.8 & 3.1 \\
SD & 3.23 & 3.19 & 1.28 \\
No. of values & 40 & 40 & 40 \\
Outlier detected & No & No & Po \\
Significance level & & & Hamilton score \\
Group 2 & Hamilton score & Outcome & Difference \\
SSRI & Initial & 20.6 & 2.2 \\
Mean & 22.8 & 1.13 & 0.61 \\
SD & 0.79 & 40 & 40 \\
No. of values & 40 & No & No \\
Outlier detected & No & & P<0.0001 \\
Significance level & & & \\
\hline
\end{tabular}

SSRI, selective serotonin reuptake inhibitors; SD, standard deviation.

depressive symptoms and their general severity, reflected by a final score, which indicates the degree of depression. The minimum score is 0 points and the maximum, 52 points. Symptoms are described as anchor points comprising between 3 and 5 possible responses to each item, with increasing severity. The score between 0 and 7 points indicates the state of normalcy, the score between 8 and 17 points represents a slight level of depression, between 18 and 24 points a moderate level of depression, and a score above 24 means a severe level of depression (16). The change in depressive symptoms from the first to the last session of therapy was assessed and bioresonance therapy was applied once a week. For the second group, the change in depressive symptoms was assessed after five weeks on antidepressant medication. The Hamilton score analysis is presented in Table III.

Statistical analysis. For statistical calculations, Graph Pad 3.6 (GraphPad Software, Inc.) was used. The Student's t-test was used to assess the differences between the means of continuous variables (expressed as mean \pm SD), while differences between nonparametric variables (expressed as median, range) were compared using the Mann-Whitney test. We interpreted all tests against a $\mathrm{P}=0.05$ significance threshold and statistical significance was considered for P-values below the significance threshold.

\section{Results}

The first group included 40 patients, having a baseline score between 12 and 24 (mean 16.9, standard deviation (SD) 3.23) on Hamilton Depression Rating Scale, with 17 items (Table III). The patients of the first group received only bioresonance therapy for five weeks, without any other type of treatment.

The second group, the SSRI group, included 40 patients having a baseline score on the Hamilton Depression Rating Scale, with 17 items between 22 and 24 (mean 22.8, SD 0.79) (Table III). The second group received either newly introduced or on-going pharmacological treatment with selective serotonin reuptake inhibitors in monotherapy for five weeks. After the applied treatment, an outcome measurement of the illness severity was performed. The evolution in Hamilton scores is presented in Fig. 1.

The differences were calculated for each patient. Group 1 obtained a higher decrease in the Hamilton score. The difference between the two data series was statistically different, based on the Student's t-test $(\mathrm{P}<0.0001)$.

\section{Discussion}

The current study aimed to identify whether the bioresonance therapy has quantifiable results in the treatment of patients with recurrent mild or moderate depressive disorder. In addition, it aimed to compare the therapy outcome with a group treated with selective serotonin reuptake inhibitors.

As a strength, all the patients were diagnosed with depression based on the DSMV criteria, assessed by a limited number of psychiatrists. Strict exclusion criteria were applied. The Hamilton questionnaires were applied by the same interview team to all the patients, in order to minimize, as much as possible, a subjective interpretation.

The use of alternative and complementary medicine has increased among patients with psychiatric disorders, as it has in the general population (17). Therefore, there is a need to inquire about and analyze the way the alternative solutions are functioning, if they are useful and what risks and benefits they may involve. However, evidence-based information is limited.

In the current model of mental illness care, widely used treatments include pharmacological medication, psychotherapeutic techniques such as psychotherapy, cognitive-behavioral therapy and also electroconvulsive therapy and transcranial magnetic stimulation $(18,19)$. Further research is needed to help identify cost-effective alternative therapies for the treatment of depression, especially for patients who refuse or no longer wish to continue pharmacological treatment with antidepressants $(16,20)$. 

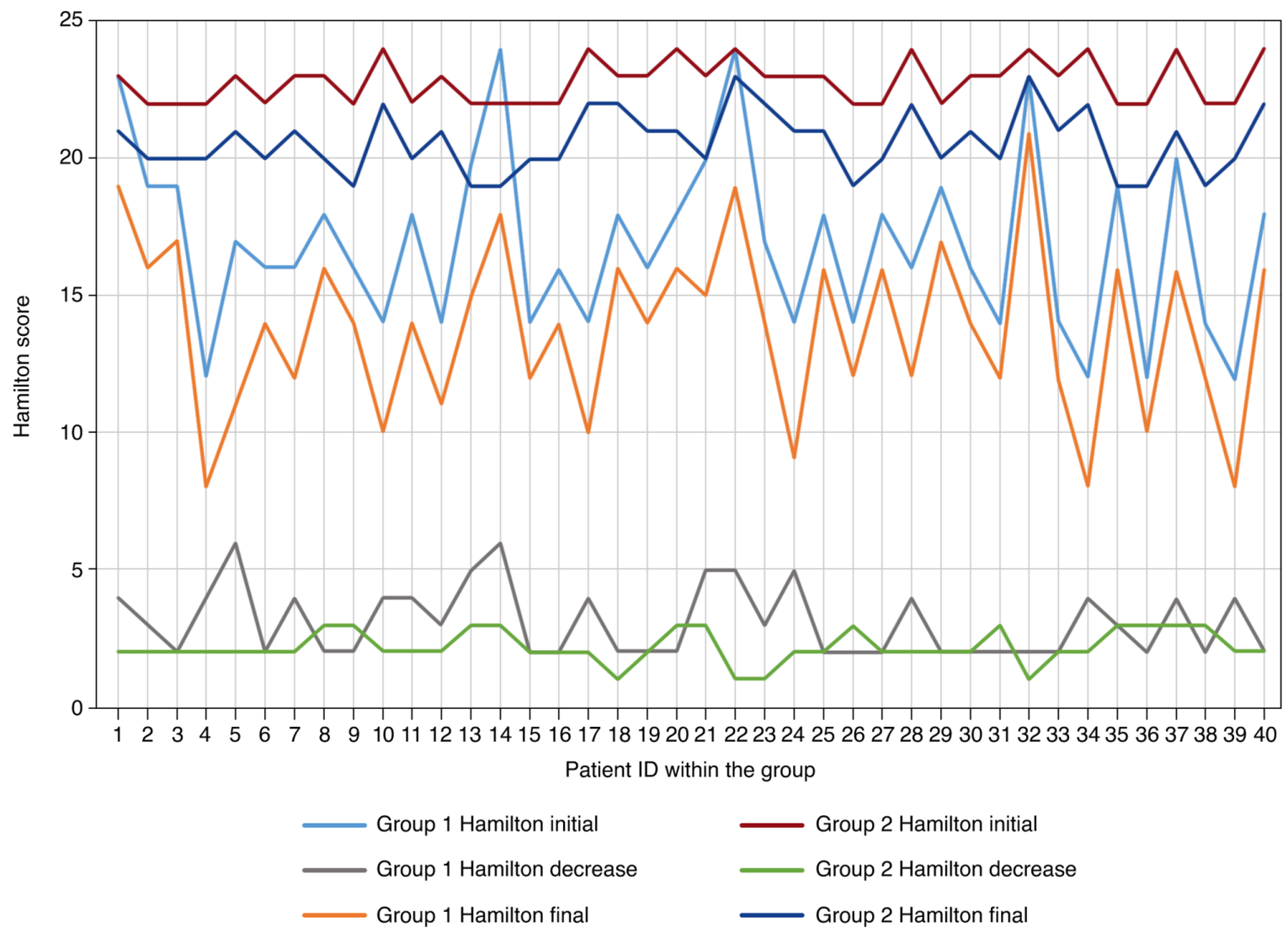

Figure 1. Evolution of the Hamilton scores.

Specialized literature presents several studies in which bioresonance therapy is successfully used in the case of various pathologies (8) To the best of our knowledge, no study thus far has conducted an objective evaluation for the effectiveness of bioresonance therapy as an alternative form of therapy in the treatment of depression.

A controlled clinical study was performed in Russia in 2018 on 60 patients, who were high-performance athletes affected by excessive physical exertion and addressed the regulation of systolic blood pressure, heart rate, as well as the reduction of stress by restoring the psycho-emotional balance. The non-placebo group had more favorable results (21).

Bioresonance therapy can significantly improve gastrointestinal disorders, as identified in a randomized controlled study performed in Germany on 20 individuals with psychosomatic diseases and gastrointestinal disorders, respectively (22).

An observational pilot study carried out in 8 patients with lymphedema and lower limb lipedema demonstrated that bioresonance therapy in lymphedema and lipedema was effective, leading to reduced edema, relieved symptoms and improved lymphatic drainage, without side effects $(19,23)$.

A German prospective controlled clinical study performed on 190 smokers has proven that Mora bioresonance therapy was effective in discontinuing smoking and presented no side effects $(8,20)$.

A study conducted in Russia analyzed the synthesis of proteins by lymphocytes from patients with rheumatoid arthritis who received bioresonance therapy. This form of therapy is used to correct energy disorders in the body's meridian system, with electromagnetic waves at a frequency range of $10-500,000 \mathrm{~Hz}$. It was identified that, after bioresonance therapy, the intensity of protein synthesis in the resting lymphocytes of the patients returned to normal $(21,24)$.

In the present study, among the patients in the first group, after applying five bioresonance therapy sessions, 4 men and 10 women improved from a moderate depressive status to a mild depressive stage. Among the study participants in the second group, both women and men remained in a moderate depressive status.

The limitations of the study are imposed by the current state of knowledge in the field of bioresonance which is less known in Romania and less studied in the world not having enough studies. In addition, the bioresonance therapy in the present study was applied with only one type of bioresonance device, but the Mora Nova device in our opinion, is one of the most powerful and complex currently available on the market. Also, the patient monitoring period was relatively short at five weeks. Surpassing the limitations of the present study can be achieved by monitoring patients for a longer period of time, using other types of bioresonance devices.

In conclusion, the study results confirmed that bioresonance therapy could improve, independently of the level of depression assessed with the Hamilton Depression Rating Scale comprising 17 items, the status of patients suffering from mild and moderate recurrent depression, who did not opt to use pharmacological treatment. 
Nevertheless, more rigorous and larger studies are recommended. At present, based on the analysis in the current study, bioresonance therapy appears promising and deserves further study.

\section{Acknowledgements}

Professional editing, linguistic and technical assistance performed by Irina Radu, Individual Service Provider (credentials: E0048/2014, Medicine-Pharmacy).

\section{Funding}

No funding was received.

\section{Availability of data and materials}

All data and materials supporting the results of the present study are available in the published article.

\section{Authors' contributions}

DM and IGG contributed to the design and conception of the study. DM, SV and CRB wrote the original draft. DM, AS and IGG participated in the data acquisition. SV and CRB contributed to statistical analysis and interpretation of the data. DM, IGG and AS worked on the part of the study related to patients. SV, AS, CRB and IGG contributed to manuscript drafting or critical revisions to the intellectual content. All authors approved the final manuscript version and agreed to be accountable for all aspects of the work. DM and IGG confirm the authenticity of all the raw data. All authors have read and approved the final manuscript.

\section{Ethics approval and consent to participate}

All patients gave their informed consent for inclusion before they participated in the study. The study was conducted in accordance with the Declaration of Helsinki and the protocol was approved by the Ethics Committee of the Mures County Hospital from Targu Mures, no. 16462/16.10.2017.

\section{Patient consent for publication}

Not applicable.

\section{Competing interests}

The authors declare that they have no competing interests.

\section{References}

1. Haller H, Anheyer D, Cramer H and Dobos G: Complementary therapies for clinical depression: An overview of systematic reviews. BMJ Open 9: e028527, 2019.

2. American Psychiatric Association: Diagnostic and statistical manual of mental disorders. 5th edition. American Psychiatric Association Press, Arlington, VA, pp155-188, 2013.

3. Rang HP, Dale MM, Ritter JM, Flower RJ and Henderson G: Rang and Dale's pharmacology. 7th edition. International Edition. British Library Cataloguing in Publication Data. Elsevier Inc., Edinburgh, pp553-583, 2012.
4. James SL, Abate D, Abate KH, Abay SM, Abbafati C, Abbasi N, Abbastabar H, Abd-Allah F, Abdela J, Abdelalim A, et al: Global, regional, and national incidence, prevalence, and years lived with disability for 354 diseases and injuries for 195 countries and territories, 1990-2017: A systematic analysis for the global burden of disease study 2017. Lancet 392: 1789-1858, 2018.

5. Wittchen HU, Jacobi F, Rehm J, Gustavsson A, Svensson M, Jönsson B, Olese J, Allgulander C, Alonso J, Faravelli C, et al: The size and burden of mental disorders and other disorders of the brain in Europe 2010. Eur Neuropsychopharmacol 21: 655-679, 2011.

6. Malhi GS and Mann JJ: Depression. Lancet 392: 2299-2312, 2018.

7. Wang PS, Aguilar-Gaxiola S, Alonso J, Angermeyer MC, Borges G, Bromet EJ, Bruffaerts R, de Girolamo G, de Graaf R, Gureje $\mathrm{O}$, et al: Use of mental health services for anxiety, mood, and substance disorders in 17 countries in the WHO world mental health surveys. Lancet 370: 841-850, 2007.

8. Pihtili A, Galle M, Cuhadaroglu C, Kilicaslan Z, Issever H, Erkan F, Cagatay T and Gulbaran Z: Evidence for the efficacy of a bioresonance method in smoking cessation: A pilot study. Forsch Komplementmed 21: 239-245, 2014.

9. Gottfried C: The new diagnostic system by Dr. Cornelissen. IMZ Akademie, pp157-171, 2007.

10. Med-Tronik GmbH: Manual of instructions. Version 3.1.3. Med-Tronik GmbH, pp72, 2020.

11. Muresan D, Salcudean A, Sabau CD, Bodo CR and Gabos Grecu I: Bioresonance therapy may treat depression. J Med Life 14: 238-242, 2021.

12. Med-Tronik GmbH: Nova MED Training\&Therapy Manual, medical technology in the 21 st century. Med-Tronik $\mathrm{GmbH}$, pp16-37, 2014.

13. Muresan D, Salcudean A, Barbu BV, Bodo CR and Gabos GI: Evaluation of an alternative depression therapy using bioresonance. J Interdiscip Med 6: 82-86, 2021.

14. O'Donnell JM, Bies RR and Shelton RC: Drug therapy of depression and anx iety disorders. Chapter 15. In: Goodman and Gilman's pharmacological basis of therapeutics, 13th edition. Brunton LL, Hilal-Dandan R, Knollmann BC (eds). McGraw-Hill, New York, NY, pp267-277, 2001.

15. Stahl SM: Selectivity of SSRIs: Individualising patient care through rational treatment choices. Int J Psychiatry Clin Pract 8 (Suppl 1): S3-S10, 2004

16. Zimmerman M, Martinez HJ, Young D, Chelminski I and Dalrymple K: Severity classification on the Hamilton depression rating scale. J Affect Disord 150: 384-388, 2013.

17. Freeman MP: Complementary and alternative medicine (CAM) Considerations for the treatment of major depressive disorder. J Clin Psychiatry 70 (Suppl 5): S4-S6, 2009.

18. Lake $\mathrm{J}$ and Turner MS: Urgent need for improved mental health care and more collaborative model of care. Perm J 21: 17-024, 2017.

19. Trifu S, Sevcenco A, Stănescu M, Drăgoi AM and Cristea BM: Efficacy of electroconvulsive therapy as a potential first-choice treatment in treatment-resistant depression (Review). Exp Ther Med 22: 1281, 2021.

20. Nguyen J: A literature review of alternative therapies for postpartum depression. Nurs Womens Health 21: 348-359, 2017.

21. Badtieva VA, Pavlov VI, Khokhlova MN and Pachina AV: The application of bioresonance therapy for the correction of the overtrained athlete syndrome. Vopr Kurortol Fizioter Lech Fiz Kult 95: 51-57, 2018 (In Russian).

22. Nienhaus J and Galle M: Placebo-controlled study of the effects of a standardized MORA bioresonance therapy on functional gastrointestinal complaints. Forsch Komplementmed 13: 28-34, 2006 (In German).

23. Elio C, Guaitolini E, Paccasassi S, Rosati N and Cavezzi: Application of microcurrents of bioresonance and transdermal delivery of active principles in lymphedema and lipedema of the lower limbs: A pilot study. G Ital Dermatol Venereol 149: 643-647, 2014.

24. Islamov BI, Funtikov VA, Bobrovskiı̌ RV and Gotovskiŭ YV: Bioresonance therapy in rheumatoid arthritis and heat shock proteins. Bull Eksp Biol Med 128: 525-528, 1999 (In Russian).

This work is licensed under a Creative Commons Attribution-NonCommercial-NoDerivatives 4.0 International (CC BY-NC-ND 4.0) License. 